\title{
Emergence of Functional Sensory Subtypes as Defined by Transient Receptor Potential Channel Expression
}

\author{
Jens Hjerling-Leffler, ${ }^{1,2}$ Mona AlQatari, ${ }^{2}$ Patrik Ernfors, ${ }^{1}$ and Martin Koltzenburg ${ }^{2}$ \\ ${ }^{1}$ Division of Molecular Neurobiology, Department of Medical Biochemistry and Biophysics, Karolinska Institutet, 17177 Stockholm, Sweden, and \\ ${ }^{2}$ University College London Institute of Child Health, University College London, London WC1N 1EH, United Kingdom
}

\begin{abstract}
The existence of heterogeneous populations of dorsal root ganglion (DRG) neurons conveying different somatosensory information is the basis for the perception of touch, temperature, and pain. A differential expression of transient receptor potential (TRP) cation channels contributes to this functional heterogeneity. However, little is known about the development of functionally diverse neuronal subpopulations. Here, we use calcium imaging of acutely dissociated mouse sensory neurons and quantitative reverse transcription PCR to show that TRP cation channels emerge in waves, with the diversification of functional groups starting at embryonic day 12.5 (E12.5) and extending well into the postnatal life. Functional responses of voltage-gated calcium channels were present in DRG neurons at E11.5 and reached adult levels by E14.5. Responses to capsaicin, menthol, and cinnamaldehyde were first seen at E12.5, E16.5, and postnatal day 0 (P0), when the mRNA for TRP cation channel, subfamily V, member 1 (TRPV1), TRP cation channel, subfamily M, member 8 (TRPM8), and TRP cation channel, subfamily A, member 1 (TRPA1), respectively, was first detected. Cold-sensitive neurons were present before the expression or functional responses of TRPM8 or TRPA1. Our data support a lineage relationship in which TRPM8-and TRPA1-expressing sensory neurons derive from the population of TRPV1-expressing neurons. The TRPA1 subpopulation of neurons emerges independently in two distinct classes of nociceptors: around birth in the peptidergic population and after P14 in the nonpeptidergic class. This indicates that neurons with similar receptive properties can be generated in different sublineages at different developmental stages. This study describes for the first time the emergence of functional subtypes of sensory neurons, providing new insight into the development of nociception and thermoreception.
\end{abstract}

Key words: TRP cation channel; functional development; dorsal root ganglion; lineage relationship; mouse; sensory neuron

\section{Introduction}

Sensory neurons in the dorsal root ganglia (DRG) are functionally, anatomically, and neurochemically diverse. Broad functional categories include mechanoreceptors, thermoreceptors, and nociceptors (Perl, 1992). Each of these groups can be further subdivided on the basis of their response to adequate stimuli and their conduction velocity, axon diameter, and myelination status (Lawson, 2002). For example, almost all thermoreceptors in rodents are unmyelinated and respond to either cold or warm stimuli, signaling the sensation of innocuous cool or warmth (Koltzenburg, 2004). Approximately three-quarters of DRG neurons are nociceptors, and in addition to strong mechanical stimuli, most are excited by noxious heat or cold and chemical irritants (Meyer et al., 2005). It is the differential expression of ion channels that lend neurons their unique functional attributes (Julius and Basbaum, 2001). How this functional diversity emerges during embryonic development is currently not known. After the

Received Aug. 27, 2006; revised Jan. 16, 2007; accepted Jan. 17, 2007.

This work was supported by the European Commission (FP6-2003-Nest-B1), The Royal Society, the International Association for the Study of Pain, the Swedish Medical Research Council, the Swedish Foundation for Strategic Research (Center of Excellence in Developmental Biology grant), and the Hedlunds Foundation.

Correspondence should be addressed to Prof. Martin Koltzenburg, University College London Institute of Child Health, 30 Guilford Street, London WC1N 1EH, UK. E-mail: m.koltzenburg@ich.ucl.ac.uk.

DOI:10.1523/JNEUROSCI.5614-06.2007

Copyright $@ 2007$ Society for Neuroscience $\quad$ 0270-6474/07/272435-09\$15.00/0 seminal discovery of the capsaicin receptor TRPV1 (Caterina et al., 1997), it has been recognized that TRP channels are the principal determinants of this specificity and have a restricted expression pattern among sensory neurons involved in thermosensation and nociception. (Clapham, 2003; Jordt et al., 2003). TRPV1 is sensitive to noxious heat, protons, and irritant vanilloids and is a characteristic marker of polymodal nociceptors (Caterina et al., 1997, 2000). TRPM8 is activated by cold stimuli, menthol, and other cooling compounds and is found in cold-sensitive thermoreceptors (McKemy et al., 2002; Peier et al., 2002). TRPA1 is activated by several pungent chemicals, including cinnamaldehyde and allyl isothiocyanate (mustard oil) (Story et al., 2003; Bandell et al., 2004; Jordt et al., 2004; Bautista et al., 2006).

Sensory neurons can also be divided on the basis of their neurochemical phenotype. In the adult, many unmyelinated neurons are labeled by the isolectin B4 (IB4) of Griffonia simplicifolia (Silverman and Kruger, 1988), which binds to a splice variant of the extracellular matrix proteoglycan versican (Bogen et al., 2005). This population arises from neurons undergoing a perinatal switch in growth factor dependency, from nerve growth factor (NGF) to GDNF (glial cell line-derived neurotrophic factor) (Molliver et al., 1997). The IB4-positive neurons are nonpeptidergic, whereas IB4-negative neurons are peptidergic nociceptive afferents that are NGF responsive and large myelinated mechanoreceptors or proprioceptors (Priestley et al., 2002) 
Here, we studied the functional development using calcium imaging of acutely dissociated sensory neurons. We used the selective agonists capsaicin, menthol, and cinnamaldehyde to activate TRPV1, TRPM8, and TRPA1, respectively, and cold stimulation. An advantage of our functional approach is the possibility to obtain a comprehensive description of the expression of many TRP channels and thermal sensitivity on a single-cell level in relation to their growth factor responsiveness.

\section{Materials and Methods}

Cell culture. Unless otherwise stated, experiments were conducted in C57/B6 mice. In some studies, CD1 mice were used. Time-mated pregnant mice were killed, and the embryos were staged (Kaufman, 1992). For each developmental stage, experiments were done on cultures from a total of five to eight separate animals on 2 or 3 different experimental days. DRGs from all cervical, thoracic, and lumbar segments were dissected in calcium- and magnesium-free HBSS and treated with papain (20 mg/ml; Sigma, St. Louis, MO) followed by a collagenase/dispase solution [10 and $12 \mathrm{mg} / \mathrm{ml}$; Worthington Biochemical (Lakewood, NJ) and Roche Diagnostics (Indianapolis, IN)] for $20 \mathrm{~min}$ at $37^{\circ} \mathrm{C}$. The ganglia were triturated using fire-polished Pasteur pipettes until single-cell suspension and then loaded onto a Percoll gradient (centrifuged for 8 $\mathrm{min}$ at $1000 \times g$; Sigma). At embryonic day 16.5 (E16.5) and earlier stages, the papain digestion and Percoll gradient were omitted. Cells were subsequently plated in a droplet of complete F-12 medium [F-12 (Invitrogen, Carlsbad, CA), 10\% horse serum (Invitrogen), and 1\% penicillin-streptomycin (Sigma)] containing laminin (30 mg/ml; Invitrogen) on a glass coverslip that had been precoated with poly-L-lysine $(0.01 \%$; Sigma) overnight. The cells were then left to adhere for $1-2 \mathrm{~h}$ before the coverslip was flooded with complete F-12. Coverslips were incubated at $37^{\circ} \mathrm{C}$ in a humidified $5 \% \mathrm{CO}_{2}$ incubator. Experiments were conducted $2-4 \mathrm{~h}$ after plating of the cells.

Calcium imaging and vital staining. For ratiometric calcium imaging, coverslips were transferred into extracellular fluid (ECF) [(in mM) 145 $\mathrm{NaCl}, 5 \mathrm{KCl}, 10 \mathrm{HEPES}, 2 \mathrm{CaCl} 2,1 \mathrm{MgCl} 2$, and $10 \mathrm{D}$-glucose] buffered to $\mathrm{pH} 7.4$ at room temperature and containing $2 \mu \mathrm{M}$ fura- $2 \mathrm{AM}$ (derived from a stock solution of $2 \mathrm{~mm}$ fura dissolved in DMSO) and $80 \mu \mathrm{M}$ Pluronic F-127 (both from Invitrogen) for $30 \mathrm{~min}$. Coverslips were subsequently placed for $10 \mathrm{~min}$ in ECF containing $10 \mu \mathrm{g} / \mathrm{ml}$ IB4 of Griffonia simplicifolia conjugated to FITC (Sigma) to allow vital staining of cells and de-esterization of fura-AM. Afterward, the coverslips were transferred to a recording chamber mounted on a Zeiss (Oberkochen, Germany) Axiovert 200 inverse microscope and viewed with appropriate filters to visualize the fluorophores. For calcium imaging, we used a $10 \times$ Fluar objective and a DCLP 410 dichroic mirror and a long-pass LP 440 emitter filter. Pairs of images were collected at intervals of 1 or $2 \mathrm{~s}$ with alternating exposure of 340 and $380 \mathrm{~nm}$ each for 50-200 ms using a Polychrome IV and an air-cooled CCD Imago Camera $(640 \times 480$ pixel resolution) that were controlled by TILLvisION 4.0 software (all from TILL Photonics, Gräfeling, Germany). This software was also used to compute ratios of fluorescent images on a pixel-by-pixel basis representing changes of intracellular calcium transients. The resulting ratios were displayed as a function of their magnitude as pseudocolor images or as kinetics over the time of the experiments. Coverslips were superfused with ECF, and drugs were applied using a gravity-driven application system with magnetic valves allowing rapid fluid exchange rates at a uniform speed of $\sim 2 \mathrm{ml} / \mathrm{min}$ in each experiment. We used a battery of stimuli that were delivered in the same sequence for all experiments. After a baseline period of $60 \mathrm{~s}$ of ECF application at room temperature, cooled ECF was applied for $30 \mathrm{~s}$ using a custom-made application system. The temperature was measured with a thermocouple, which was positioned in the stream of the applied fluid just outside of the visual field on the opposite side of the application device. The cooling was adjusted so that a nadir of $5^{\circ} \mathrm{C}$ was reached at the end of the stimulus, after which temperature returned to baseline. Low humidity in the room and thorough cleaning of the outside of the recording chamber prevented fogging during the stimulus duration. Cold stimuli were followed by a sequence of chemical stimuli consisting of $250 \mu \mathrm{M}$ of the TRPM 8 agonist menthol for $10 \mathrm{~s}, 100 \mu \mathrm{M}$ of the TRPA1 agonist cinnamaldehyde (Sigma) for $60 \mathrm{~s}$, $1 \mu \mathrm{M}$ of the TRPV1 agonist capsaicin for $4 \mathrm{~s}$, and 50 or $100 \mathrm{~mm} \mathrm{KCl}$ for $6 \mathrm{~s}$, allowing a washout of at least $60 \mathrm{~s}$ between the end and onset of each stimulus. In some experiments, we used $100 \mu \mathrm{M}$ mustard oil for $60 \mathrm{~s}$ between the applications of cinnamaldehyde and capsaicin. Stock solutions of TRP channel agonists were made up in ethanol and diluted freshly to the concentrations indicated with a final ethanol concentration of 0.01 or $0.005 \%$. Recently, we showed that $18 \pm 4 \%$ of adult DRG neurons responded to mustard oil, whereas cinnamaldehyde excited $7 \pm$ $4 \%(n=566)$ (Munns et al., 2007). However, studies of adult mice lacking TRPA1 have found a significantly reduced but not abolished response to mustard oil, suggesting that this compound is less selective and appears to excite sensory neurons by alternative modes of action (Kwan et al., 2006). Kinetics of individual cells were analyzed, and a response was scored if there was a time-locked increase of the ratio of $30 \%$ from baseline.

Immunohistochemistry. Coverslips were fixed in $4 \%$ paraformaldehyde (Merck, Whitehouse Station, NJ) at room temperature for $5 \mathrm{~min}$, permeabilized, and blocked using 5\% donkey serum (Millipore, Billerica, MA) in dilution buffer consisting of PBS with $0.5 \mathrm{M} \mathrm{NaCl}, 2.5 \%$ BSA (Sigma), and $0.3 \%$ Tween 20 (Merck). Incubation with primary antibodies against $\beta$ III-tubulin (Tuj1; mouse; 1:250; Millipore) or against mouse TRPV1 (RA14113, rabbit, 1:500; Neuromics, Edina, MN) (overnight) and with FITC-conjugated IB4 $(10 \mu \mathrm{g} / \mathrm{ml})$ at $4^{\circ} \mathrm{C}$ (overnight) was followed by species- and isotype-specific fluorescent antibodies [cyanine 2 (Cy2)- or Cy3-conjugated anti-mouse donkey antibodies, 1:400 (Jackson ImmunoResearch, West Grove, PA); or Alexa594-conjugated anti-rabbit donkey antibodies, 1:1000 (Invitrogen)] for $1 \mathrm{~h}$ at room temperature in dilution buffer. Nuclear staining was performed by treatment with propidium iodide ( $10 \mu \mathrm{g} / \mathrm{ml}$; Sigma) in PBS for $15 \mathrm{~min}$ at room temperature. Pictures were taken using a Zeiss LSM 510 confocal microscope or an Axiovert 200.

Quantitative reverse transcription-PCR. Total RNA was extracted from quickly dissected DRGs of all levels of E11.5 to adult using the Purescript RNA Purification kit (Flowgen, Nottingham, UK) in four separate animals at each time point. In another set of experiments on adult DRG cells, RNA was extracted after the Percoll step or after $2 \mathrm{~h}$ in culture. Samples were DNase treated with the Turbo DNA-free kit (Ambion, Huntingdon, UK) and quantified on a NanoDrop 1000 spectrophotometer (NanoDrop Technologies, Wilmington, DE). RNA quality and integrity were assessed on an RNA Nanochip and Bioanalyzer 2100 (Agilent, Palo Alto, CA). All samples had an RNA integrity value of 8.5-9.5. One hundred nanograms of RNA were reverse transcribed using the iScript kit (BioRad, Hemel Hempstead, UK) and used for real-time PCR using SYBR green (Bio-Rad) and specific primers for ubiquitin C-terminal hydrolase isozyme L1 (UCHL1), glyceraldehyde-3-phosphate dehydrogenase (GAPDH), TRPV1, TRPM8, or TRPA1 on a Smart Cycler (Cepheid, Sunnyvale, CA). All assays showed a linear correlation between cycle threshold and amplicon in serial dilution experiments with PCR efficiency of $92-97 \%$. Reaction products gave single peaks on melt curve analysis and single bands of expected size (UCHL1, 117 bp; GAPDH, 81 bp; TRPV1, 120 bp; TRPM8, 190 bp; TRPA1, 107 bp) on a 2\% agarose gel. PCR products were sequenced from three separate reactions to verify that the correct transcript had been amplified. Relative quantification was performed using the $\Delta \Delta$ method and expressed in relation to the housekeeping gene GAPDH as a reference (Pfaffl et al., 2002) and percentage of adult values or as $\Delta$ difference in cycle thresholds to UCHL1.

Data presentation and statistical tests. All values are expressed as arithmetic mean and SEM of groups of individual animals or individual cultures (when tissues were pooled from several animals of the same age in calcium-imaging experiments). One-way ANOVA with Fisher's least significant difference post hoc test was used for statistical comparison.

\section{Results}

\section{Initial differentiation of sensory neurons}

We used acutely dissociated DRG cells from all spinal segments. The same preparation was used for the immunohistochemical and molecular studies. At E11.5, neural crest cells have completed migration and are committed to the sensory lineage. However, in 


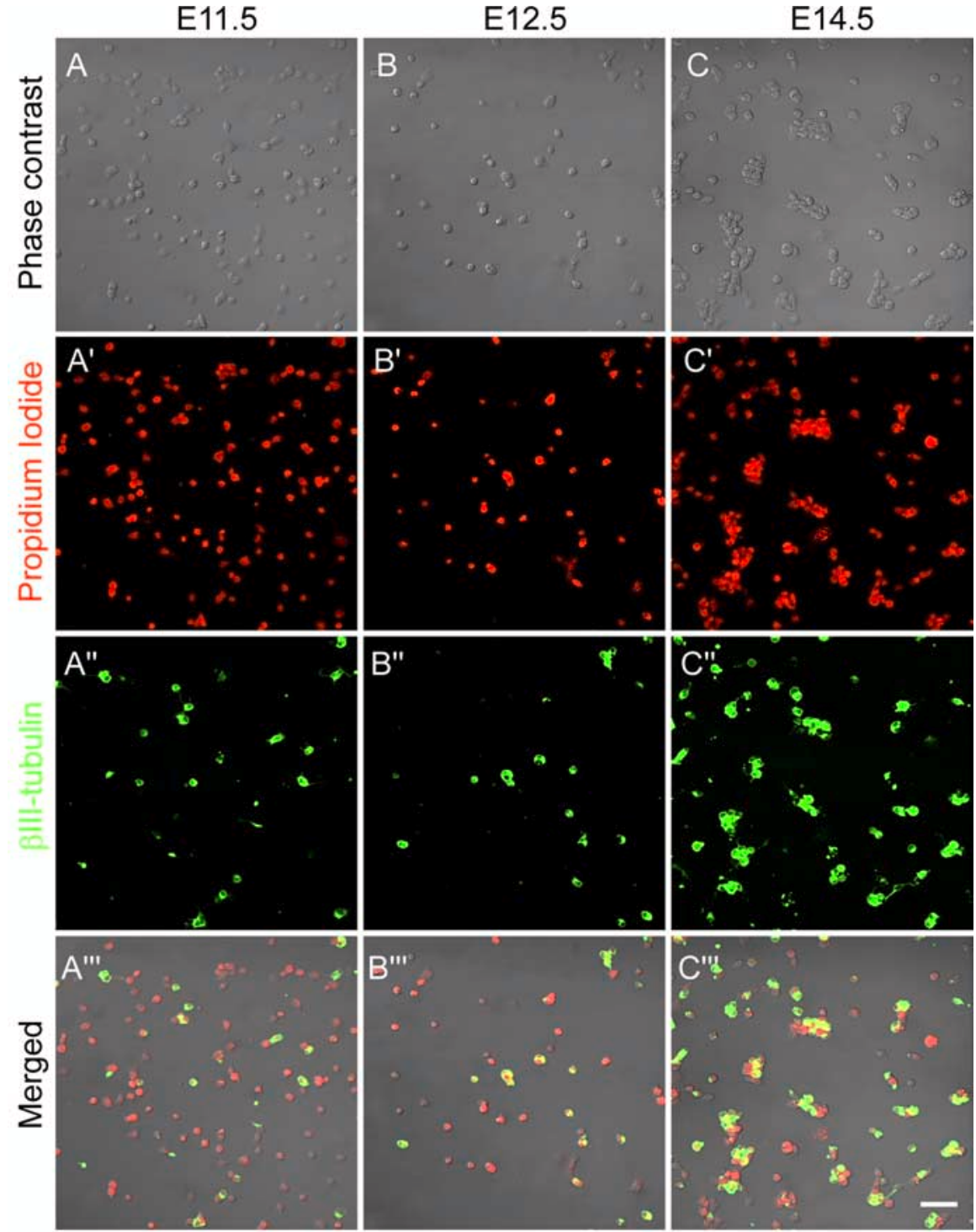

D

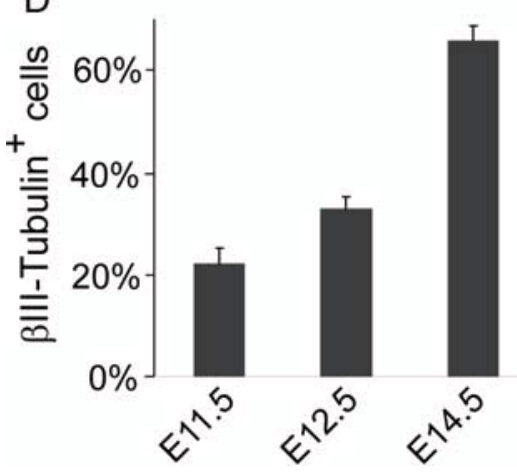

E

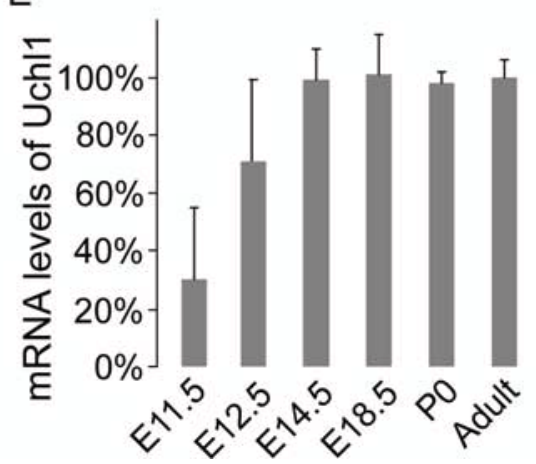

Figure 1. Cultured DRG cells from all rostrocaudal levels at E11.5, E12.5, and E14.5 stained with the general nuclear marker propidium iodide $\left(\boldsymbol{A}^{\prime}-\boldsymbol{C}^{\prime}\right.$, red) and for the neuronal marker $\beta$ III-tubulin $\left(\boldsymbol{A}^{\prime \prime}-\boldsymbol{C}^{\prime \prime}\right.$, green; $\boldsymbol{A}^{\prime \prime \prime}-\boldsymbol{C}^{\prime \prime \prime}$, merged $)$, showing the gradual increase in neuronal differentiation. Scale bar, $50 \mu \mathrm{m}$. $\boldsymbol{D}$, Quantification of $\boldsymbol{A}-\boldsymbol{C}(n=3)$. $\boldsymbol{E}$, mRNA levels of the neuronal marker UCHL1, normalized against the adult.

cultures taken from E11.5 or E12.5 embryos, it is impossible to unequivocally distinguish neuronal precursors from differentiated sensory neurons on the basis of their morphology. Therefore, we used immunohistochemistry for the early neuronal marker $\beta$ III-tubulin (Fig. $1 A-C$ ). At E11.5, $>20 \%$ of the cells were $\beta$ III-tubulin, and this increased to $32.9 \pm 2.3 \%$ by E12.5 and further to $65.8 \pm 2.8 \%$ by E14.5 ( $p \ll 0.001)$. Another early neuronal marker for peripheral sensory neurons is UCHL1. We measured mRNA expression by quantitative reverse transcription-PCR (qrtPCR) When normalized to adult levels, there was a significant increase from E11.5 to E14.5 (Fig. $1 E)(p<0.01)$ but no significant change at subsequent developmental ages $(p>0.5)$. Thus, both independent measures agree that the developmental expression of neuron-specific markers reach adult levels by E14.5. We also studied the functional appearance of voltage-gated calcium channels (VGCCs), which are present in DRG neurons but not in nonneuronal DRG cells. We found that depolarizing shifts of the membrane potential by application of $50 \mathrm{~mm} \mathrm{KCl} \mathrm{evoked} \mathrm{sharp}$ calcium transients from E11.5 onward (Fig. 2). Increasing the $\mathrm{KCl}$ concentration to $100 \mathrm{~mm}$ did not activate cells that were unresponsive to $50 \mathrm{~mm}$. There was a gradual significant increase of responses in the cultures from $5.0 \pm 0.02 \%$ of the cells at E11.5 and $22.5 \pm 0.1 \%$ at E12.5 $(p<$ $0.05)$. At E14.5, $77.4 \pm 0.4 \%$ of the cells with morphological features of neurons showed this response, which is similar to cultures at later stages.

Development of TRP channel agonist and cold reception by sensory neurons We did not observe any calcium transients in response to TRP channel agonists in cells that were unresponsive to $\mathrm{KCl}$. At E11.5, 1 of $40 \mathrm{KCl}$-responsive cells showed a small capsaicin response, and 2 others responded to cold (Fig. 2). At E12.5, $5.2 \pm$ $4.2 \%$ of neurons ( $n=590,4$ animals) identified by their VGCC expression responded to capsaicin, which corresponds to $\sim 1 \%$ of all cells in these cultures. Thus, the low percentage of capsaicin responses cannot be attributed to lack of amplification of a capsaicin-induced depolarizing response through VGCC. At E14.5, there was a dramatic increase of the percentage of capsaicin-sensitive neurons to $64.0 \pm$ $8.4 \%$, followed by a decline at postnatal stages to $\sim 40 \%$ (Fig. $3 A$ ). A similar time course was also seen for cold-sensitive cells with a peak at E18.5 with $45.7 \pm 5.0 \%$ and $\sim 20 \%$ in the adult animal. Menthol evoked rapid time-locked increases and decreases of the intracellular calcium concentration. Responses $(0.2 \pm 0.2 \%)$ were first observed at E16.5, but over the following days, the number of cells increased [2.9 \pm $1.0 \%$ at E18.5 and $4.8 \pm 1.2 \%$ at postnatal day $0(\mathrm{P} 0)]$, and at $\mathrm{P} 7$, the amount of cells was similar to that of the adult animal. In contrast to capsaicin and menthol, cinnamaldehyde often evoked 


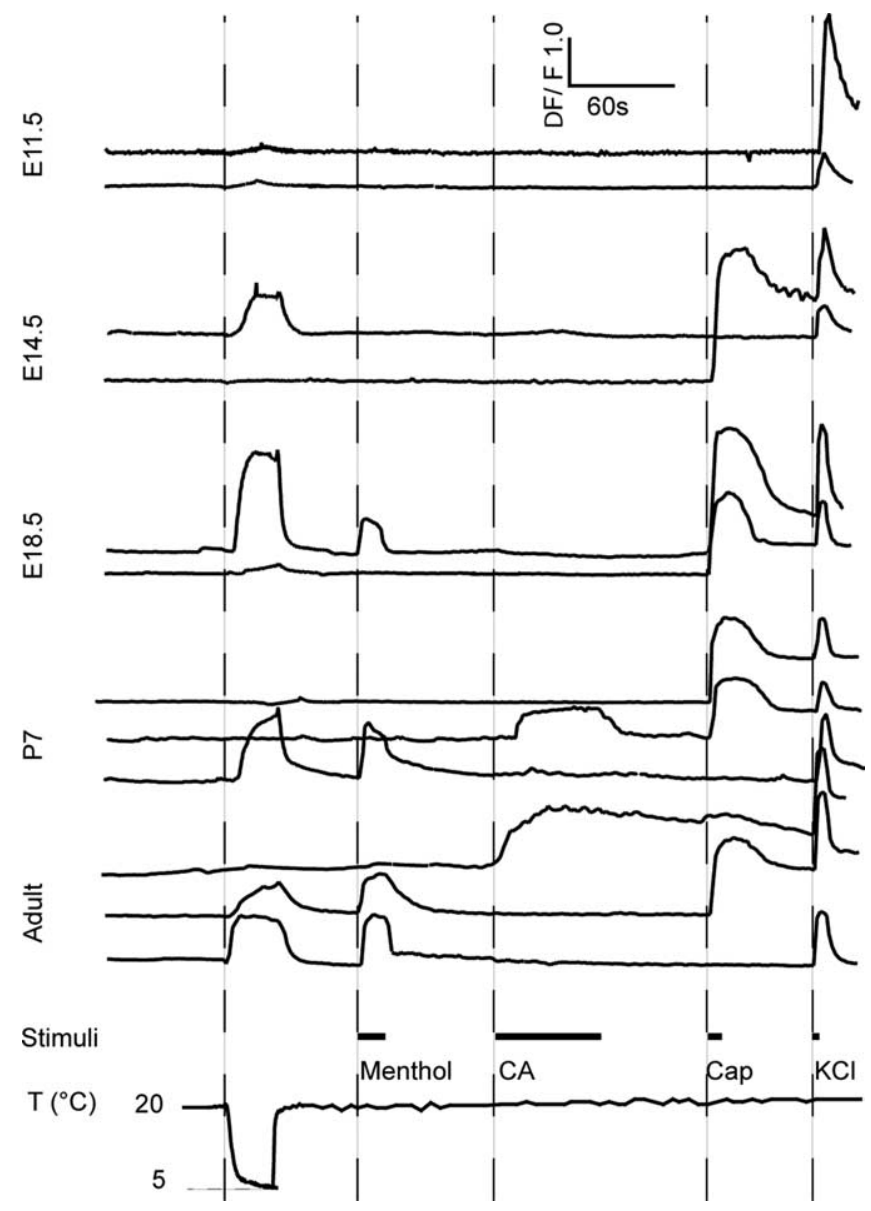

Figure 2. Representative recordings of calcium transients in DRG neurons at different developmental stages in response to cold, TRP channel agonists, and KCI. CA, Cinnamaldehyde; Cap, capsaicin; $\mathrm{T}$, temperature.

responses with a delay of several seconds, and the increase of the kinetic curve to the peak ratio was slower. Unexpectedly, the responses to cinnamaldehyde were not seen until birth. The first few cells were observed at P0 in two of five animals $(0.6 \pm 0.5 \%)$, whereas the first consistent response were observed at P7 (5.0 \pm $1.2 \%$ ), indicating that functional TRPA1 appears in the first postnatal week (Fig. 3A). Studies in CD1 mice (E16.5, 375 neurons, three mice; E18.5, 371 neurons, four mice; P0, 252 neurons, three mice; adult, 320 neurons, five mice) confirmed the sequence of events of the emergence of TRP channels found in the C57/B6 strain. There was a large proportion of capsaicin-sensitive neurons at E16.5, when menthol sensitivity emerged. No responses to cinnamaldehyde or mustard oil were detected prenatally in CD 1 mice, and $<1 \%$ of neurons responded to either stimulus at P0.

We used qrtPCR to measure the corresponding TRP gene expression. This showed that the appearance of mRNA of TRPV1, TRPM8, or TRPA1 was closely correlated with the appearance of functional responses. TRPV1 was first detected at E12.5, TRPM8 was first detected at E16.5, and TRPA1 was first detected at P0 (Fig. 3B). Quantification of these mRNA levels (Fig. 3C-E) showed a fourfold relative increase from the time when the mRNA was first detected to the next time point, which correlated with the substantial increase in the functional responses. Thus, using two independent outcome measures, we have determined that there are sequential waves of TRP channel expression and that the period of emergence of the different cell
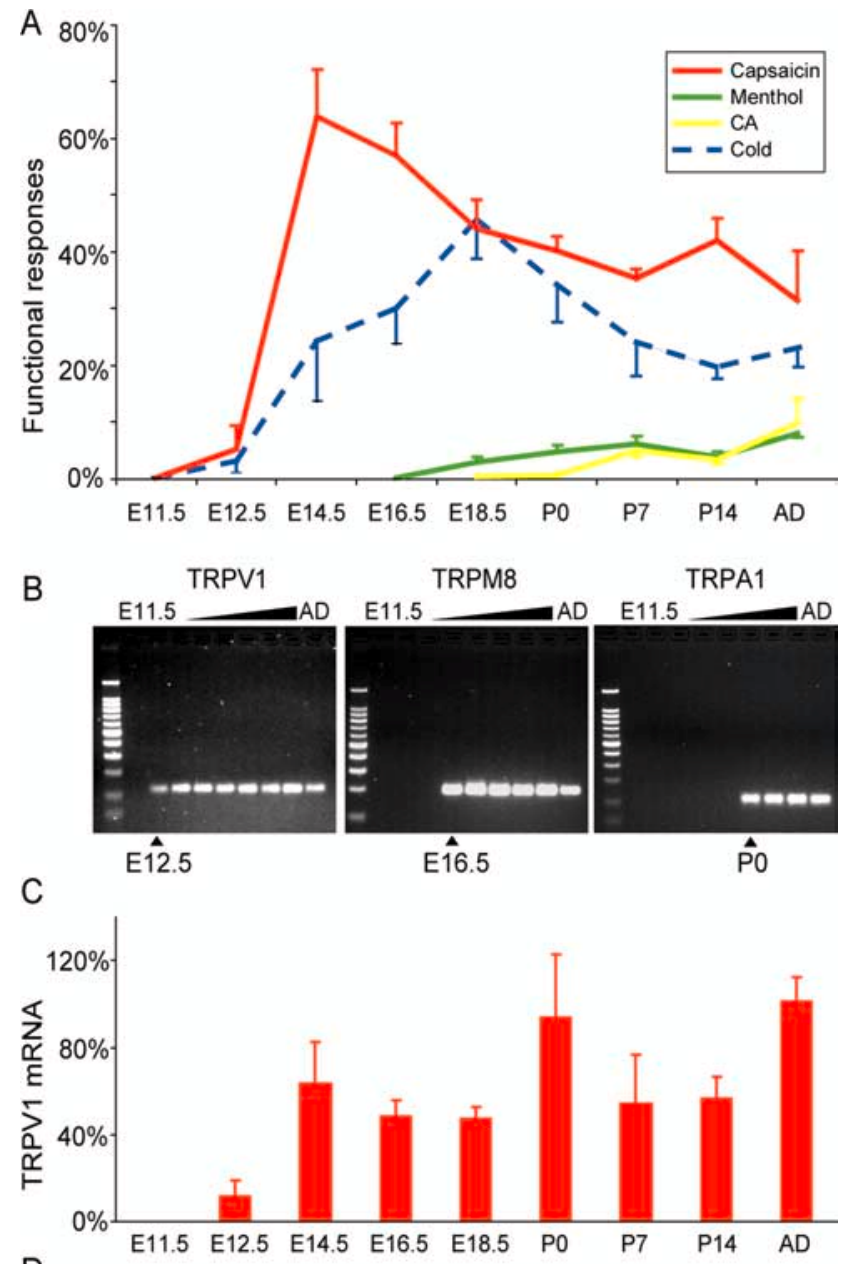

D

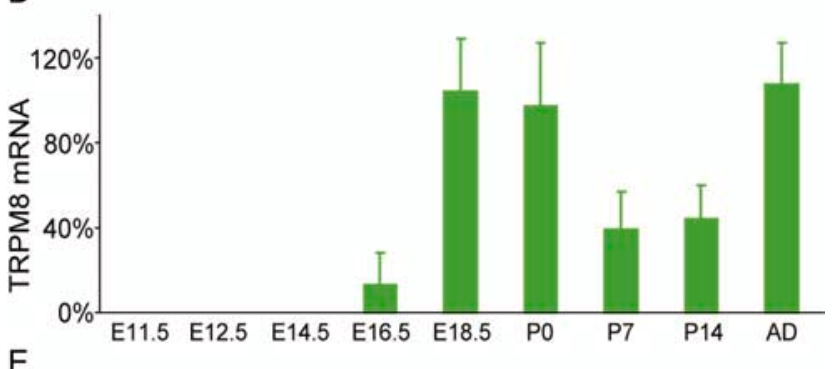

E

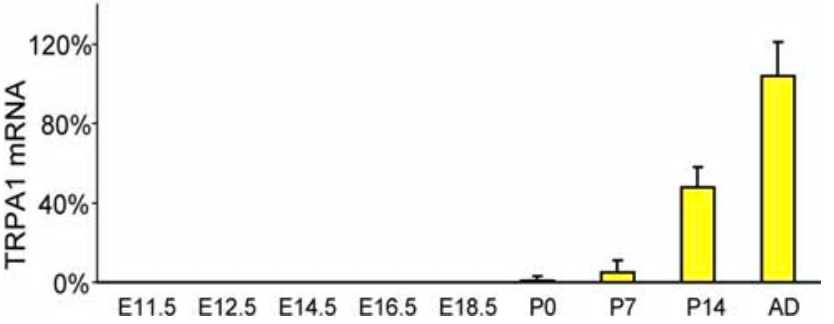

Figure 3. A, Proportion of DRG neurons responding to TRP channel agonists and cold stimulus. $\boldsymbol{B}$, Reverse transcription-PCR gels of TRPV1, TRPM8, and TRPA1 illustrating the onset of mRNA expression in the developmental stages $(n=5-8)$. The onset of functional responses coincides with the onset of mRNA. $C-E$, Quantitative reverse transcription-PCR $(n=4)$ for TRPV1 (C), TRPM8 (D), and TRPA1 (E). AD, Adult; CA, cinnamaldehyde.

types spans from early embryonic stages to the first postnatal weeks in the adolescent mouse.

We also used qrtPCR to determine that there were no significant changes of mRNA levels for TRPV1, TRPM8, or TRPA1 
A

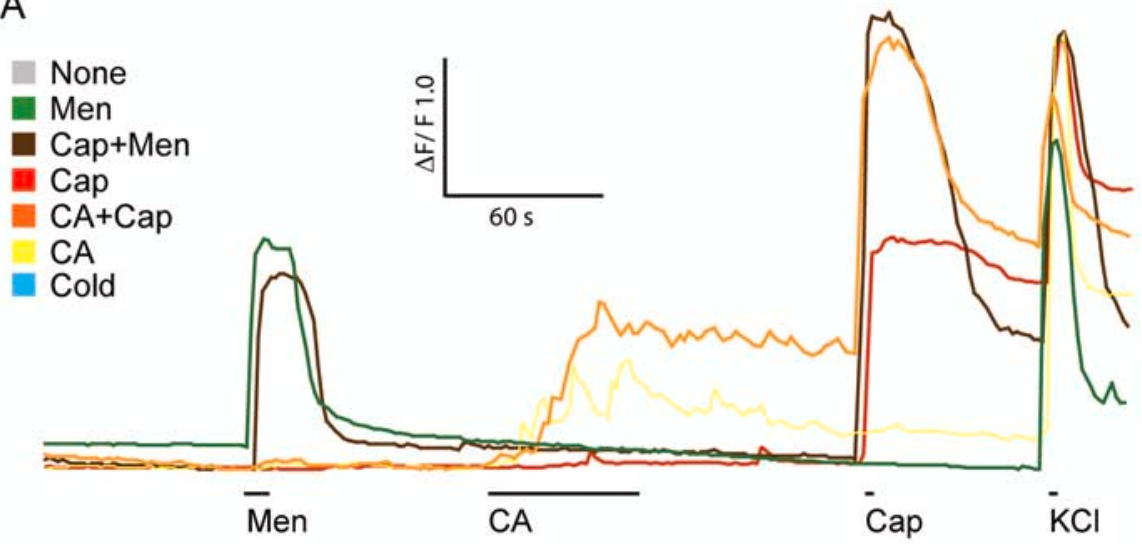

B

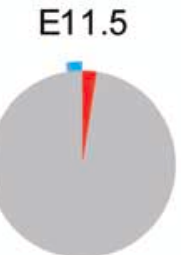

E16.5

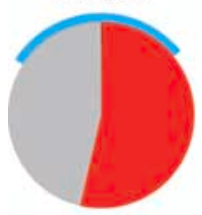

P7

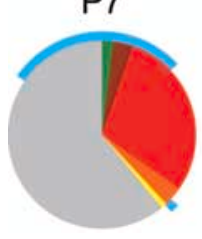

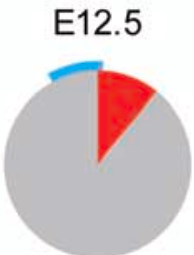

E18.5
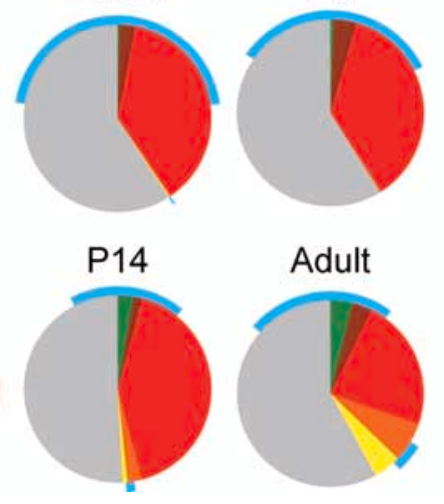

E14.5

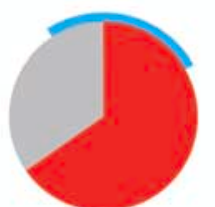

P0

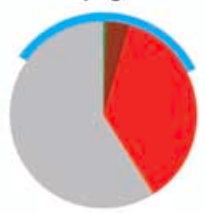

Adult
C

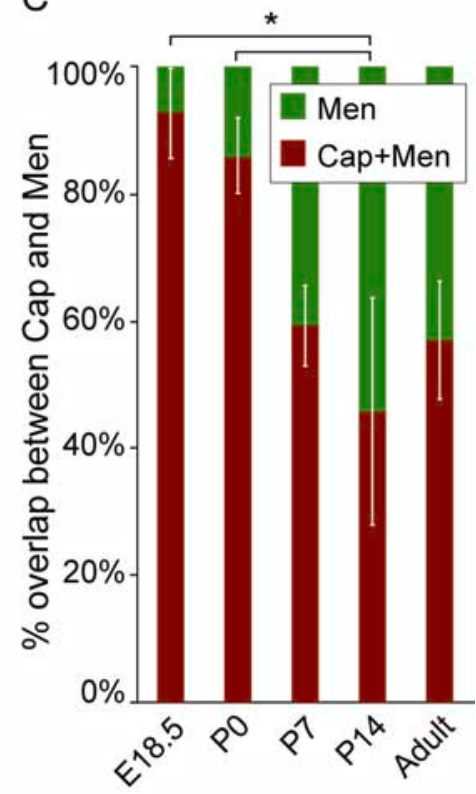

Figure 4. $\quad \boldsymbol{A}$, Representative $\mathrm{Ca}^{2+}$ transients in response to TRP channel agonists in different sensory neuron subtypes. $\boldsymbol{B}$, Pie charts showing the distribution and overlap between the different responses during the developmental stages. $\boldsymbol{C}$, Plot of the menthol-responsive cells and their response to capsaicin, showing a significant loss of capsaicin sensitivity between stages P0 and P14. ${ }^{*} p<0.05$. CA, Cinnamaldehyde; Cap, capsaicin; Men, menthol.

when comparing dissected whole DRG, freshly dissociated DRG cells (before plating), and DRG cells after $2 \mathrm{~h}$ in culture (supplemental Fig. 1, available at www.jneurosci.org as supplemental material).

\section{Functional subtypes of nociceptors}

In adult rodents, DRG neurons often respond to more than one TRP channel agonist, and we therefore studied this overlap during development. Figures 2 and $4 \mathrm{~A}$ show representative responses for different neuronal subpopulations. Except for a few cells responding to menthol at E16.5, there are only capsaicinresponsive cells before E18.5. Interestingly, capsaicin-responsive cells were not responsive to cold before E14.5. At no time of development did we observe a functional response to menthol and cinnamaldehyde in the same cell, indicating that TRPM8 and TRPA1 are expressed by different neurons (Fig. $4 B$ ). As expected, almost all TRPM8 neurons responded to cold, but many cold cells did not respond to menthol. Interestingly, in the early stages of development, the responses to menthol overlapped to a large extent with those of capsaicin (Fig. 4C). At E18.5 and P0,

$92.9 \pm 7.1$ and $86.0 \pm 5.8 \%$, respectively, of the menthol-sensitive neurons were capsaicin-sensitive, whereas at P14, it was significantly lower $(p<0.05)$, only $45.8 \pm$ $17.8 \%$.

\section{TRP channel expression in IB4-positive} and -negative neurons

Combining calcium imaging with vital IB4 staining allowed us to study the developmental expression of TRP channels in subpopulations of sensory neurons. IB4 binding was first observed at E18.5 (Fig. $5 A$ ). The percentage of IB4-positive neurons increased strongly at birth and continued to increase after birth, reaching $44 \pm 4 \%$ in the adult animal (Fig. $5 B$ ), in accordance with previous data showing the onset of IB4 binding before the switch from trkA (tyrosine receptor kinase A) to c-ret (Molliver and Snider, 1997). In the IB4-positive population, cells with menthol responses were virtually absent, and there was less coldresponsive neurons than in the IB4negative population (Fig. $5 C$ ).

Interestingly, in our functional studies we observed that over one-third of the IB4-positive neurons were capsaicin sensitive. In contrast, immunohistochemical studies in tissue sections have found that in the mouse only $5 \%$ of IB4-stained cell profiles are immunoreactive for TRPV1 (Zwick et al., 2002; Woodbury et al., 2004). We therefore performed double staining of neurons that were cultured for $2 \mathrm{~h}$ and found that $8 \pm 3 \%$ (408 cells; five animals) of IB4-positive cells were immunoreactive for TRPV1. Thus, the immunohistochemical studies together with the qrtPCR data agree that there is no upregulation of TRPV1 during shortterm culture. Furthermore, our calcium-imaging data are in line with patch-clamp recordings, which have shown that $\sim 30-40 \%$ of IB4-positive neurons in the mouse show capsaicin-mediated inward currents (Dirajlal et al., 2003; Leffler et al., 2006), which are absent in mice lacking TRPV1 (Caterina et al., 2000). Together, this suggests that TRPV1 immunostaining underestimates the number of capsaicin-sensitive neurons.

An interesting developmental difference between the IB4positive and -negative population was the loss of capsaicin responsiveness during development. Although the proportion of capsaicin-sensitive cells decreased significantly between P0 and adult $(p<0.05)$ in the IB4-negative population, there did not seem to be a corresponding loss among the IB4-positive neurons $(p>0.5)$ (Fig. 5C).

Moreover, there was a differential development of cinnamaldehyde expression among IB4-positive and -negative populations. In the IB4-positive population, the majority of cinnamaldehyde responses developed between P14 and adulthood (Fig. $5 D)(p<0.05)$. In contrast, the major emergence of TRPA1 expression in the IB4-negative occurred between P0 and P7. 


\section{Discussion}

In the present study, we describe for the first time the emergence of different functional subtypes of sensory neurons that transduce responses from a variety of stimuli. We show a gradual maturation of sensory neurons starting shortly after migrating neural crest cells become committed to the sensory lineage and extending well into postnatal life. Although it is tacitly assumed that neurons assume their functional specificity shortly after exiting the final mitosis, our results show that the specification of the functional identity of nociceptors and thermoreceptors occurs much later during embryonic development.

\section{Early sensory neuron differentiation}

Development of the nervous system occurs in a rostrocaudal manner, with events in the mouse lumbar region lagging behind those in the cervical region by $\sim 20 \mathrm{~h}$. The birth of sensory neuron is defined by the exit from cell cycle and has been studied using tritiated thymidine incorporation techniques (Lawson and Biscoe, 1979). Most DRG neurons go through their last cell division between E11 and E12 in the mouse, and the last neurons are born by E14. This is in accordance with the increase of the percentage of $\beta$ III-tubulinpositive cells and the relative mRNA levels of UCHL1. At E11.5, more than four times as many cells stain for the neuronal marker $\beta$ III-tubulin as show a functional expression of VGCC. However, by E12.5, these numbers have become similar, suggesting that expression of VGCC occurs with a time lag in differentiating dorsal root ganglion neurons similar to that in differentiating PC12 cells (Bouron et al., 1999). At E14.5 onward, it was also possible to distinguish neuronal cells from non-neuronal cells by their morphology. At this time point, the differentiation into glia has started, and non-neuronal cells on the coverslip will mostly be either glial cells or their progenitors rather than neuronal precursors (Lawson and Biscoe, 1979; Aquino et al., 2006). Other channels expressed specifically by sensory (more specifically nociceptive) neurons are sodium channels. The embryonic expression of voltage-gated sodium channels $\mathrm{Na}_{\mathrm{v}} 1.8$ and $\mathrm{Na}_{\mathrm{v}} 1.9$ in lumbar DRGs of the rat begins at E15 and E17 (Felts et al., 1997; Benn et al., 2001), which would correspond to E13.5 and E15.5 in the mouse, suggesting a gradual acquisition of ion channels with VGCCs coming on before $\mathrm{Na}_{\mathrm{v}} 1.8$ and $\mathrm{Na}_{\mathrm{v}} 1.9$.

\section{Sequential emergence of receptive properties}

Many studies show that the molecular specification of sensory neuron subtypes studied by transcription factor or growth factor

A

B

C
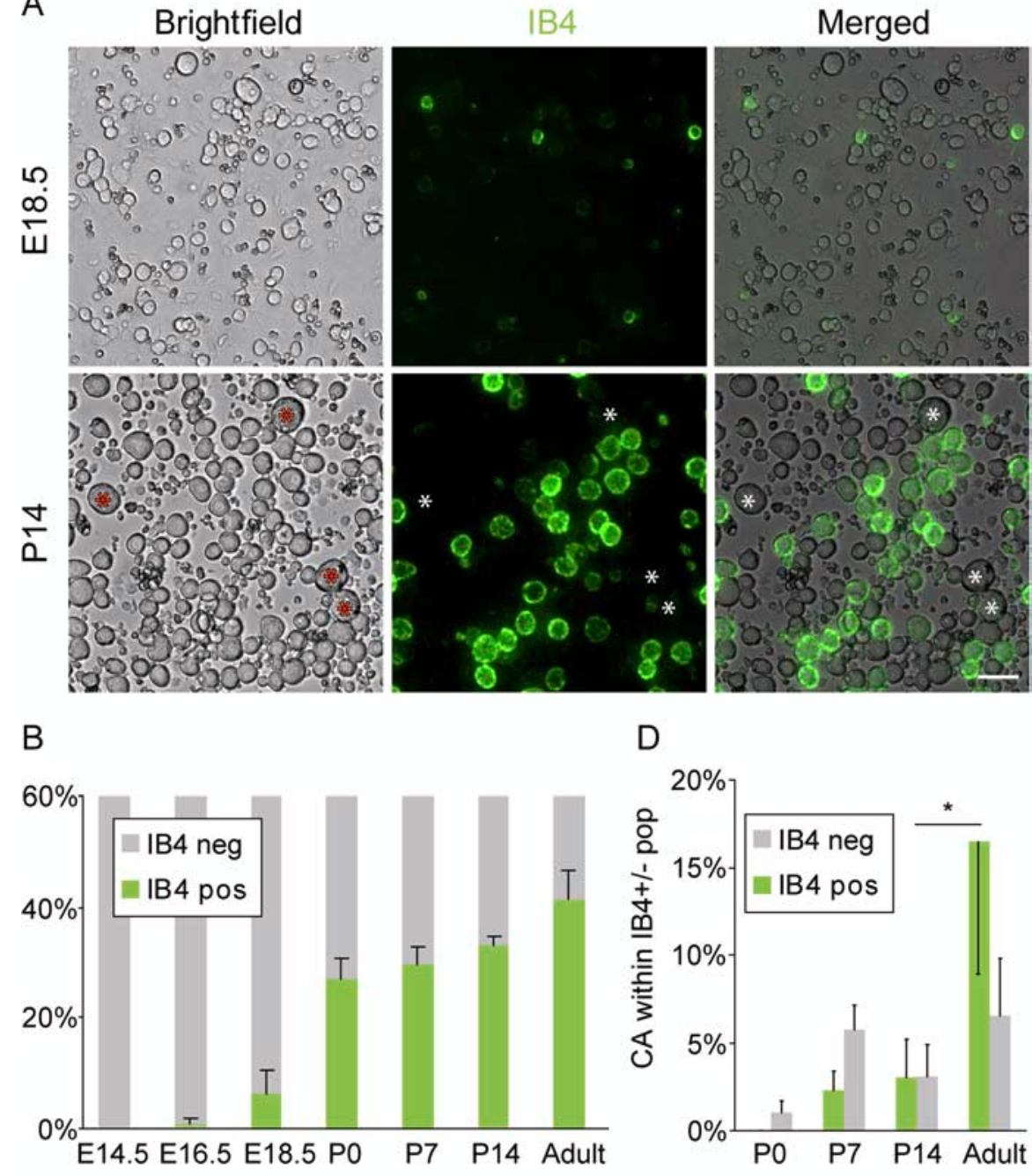

D
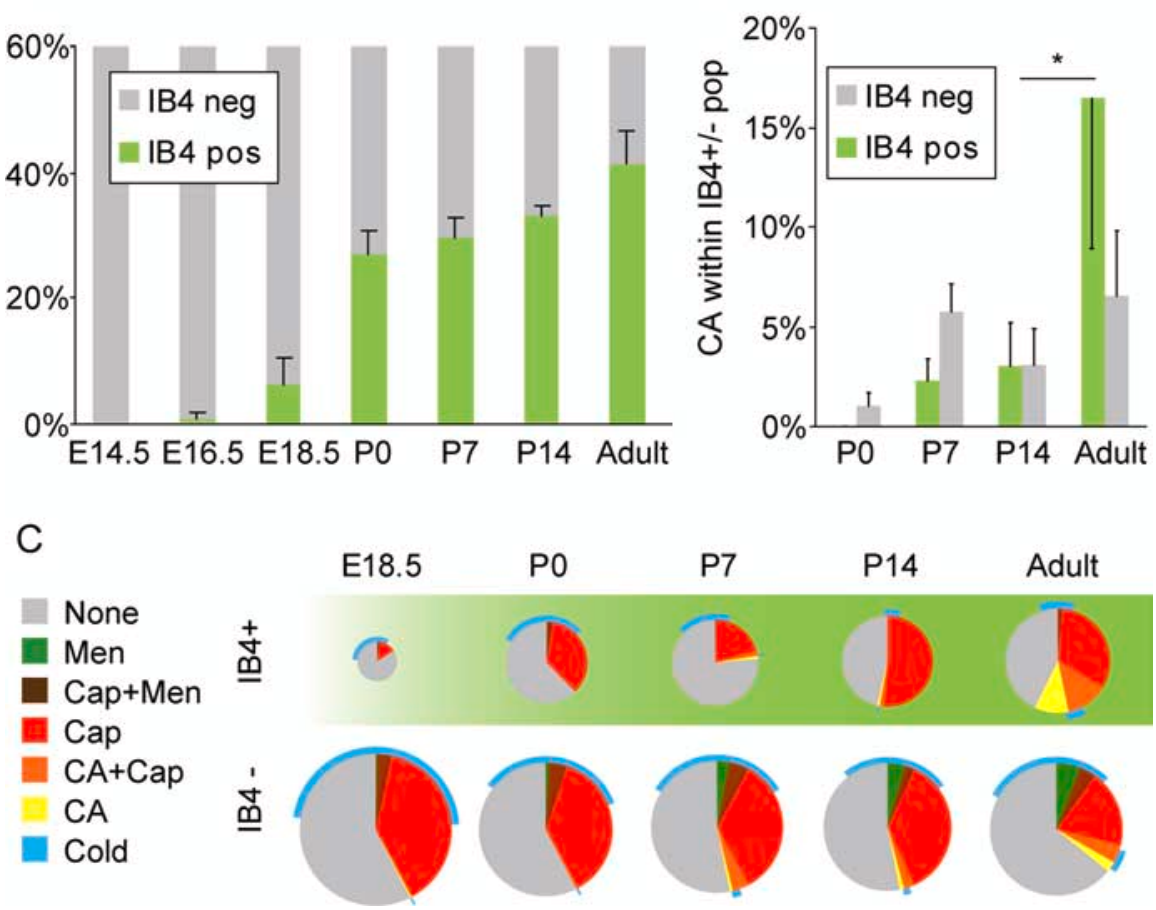

Figure 5. $\quad \boldsymbol{A}$, Vital staining with isolectin B4 (IB4; green) of dissociated DRG neurons from stages E18.5 and P14, showing preferential staining of small-diameter neurons and lack of staining in large-diameter neurons (asterisks). Scale bar, $50 \mu \mathrm{m} . \boldsymbol{B}$, Percentage of IB4-binding neurons at different developmental stages. C, Pie chart showing the functional responsiveness in relation to IB4 staining. Note the virtual absence of menthol responses and the late onset of a large proportion of cinnamaldehyderesponsive neurons within the IB4 ${ }^{+}$population. $D$, Graph showing the amount of cinnamaldehyde-responsive cells in relation to IB4 staining, showing a significant increase of cinnamaldehyde sensitivity between P14 and adult in the IB4-positive population. CA, Cinnamaldehyde; Cap, capsaicin; Men, menthol; neg, negative; pos, positive.

receptor expression studies occurs early in development. Large mechanoreceptive and small nociceptive neurons can be identified as early as E10 to E12 in the mouse (Snider, 1994; Lin et al., 1998; Inoue et al., 2002; Marmigere et al., 2006). TRP agonists excite only thin myelinated or unmyelinated sensory neurons (N. Vastani, K. Maurer, and M. Koltzenburg, unpublished observations). The early onset of capsaicin reactivity and TRPV1 expression at $\sim$ E12.5-E13.5 therefore suggests a $1 \mathrm{~d}$ delay from the birth 
of the neuron to the first functional determinant of its identity. Our data correspond with histological studies, which have shown the appearance of TRPV1 protein around this time (Tamura et al., 2005; Funakoshi et al., 2006). The peak in the functional response to capsaicin at E14.5 is accompanied by increased levels of TRPV1 mRNA. We do not know whether the subsequent decrease in percentage comes from a downregulation of TRPV1 in healthy cells or if disproportionately large numbers of TRPV1expressing neurons die during the period of programmed cell death. In the absence of data supporting such a biased loss (Buchman and Davies, 1993), we have to assume a downregulation of TRPV1 during development.

Robust cold responses were present in neurons from E12.5, well before the onset of TRPM8 and TRPA1, suggesting there are additional cold transduction pathways (Reid, 2005). All neurons acquiring menthol sensitivity, but only a minority of neurons becoming cinnamaldehyde sensitive, are also activated by cold. Our data therefore add to a growing body of evidence questioning the role of TRPA1 in cold sensation (Jordt et al., 2004; Reid, 2005; Bautista et al., 2006).

A perhaps surprising finding of the study was the late developmental emergence of TRPA1. Its ligand, mustard oil, has been used to study nociception in neonatal rats. These studies have shown that mustard oil-evoked flexion reflexes are weak or absent at birth (Fitzgerald and Gibson, 1984) and that mustard oil induction of c-fos in neurons of the dorsal horn of the spinal cord was absent (Williams et al., 1990). Whereas this previous work was interpreted as evidence for an immaturity of central synapses, our data argue that the lack of TRPA1 expression is responsible for the weak or absent effects of mustard oil at birth.

In situ hybridization and immunohistochemical studies have suggested the complete lack of overlap between TRPM8 and TRPV1 and that TRPA1-expressing neurons are mostly contained in the TRPV1-positive neurons (Peier et al., 2002; Story et al., 2003; Bautista et al., 2005; Kobayashi et al., 2005). This is clearly not in accordance with the present functional data in the adult mouse, in which $44.7 \%$ of the menthol-responsive population also responded to capsaicin, whereas a substantial number of cinnamaldehyde-responsive neurons did not. Our results in the adult are, however, in agreement with other functional studies, which have shown similar overlap between menthol and capsaicin sensitivity (McKemy et al., 2002) and a population of mustard oil-sensitive neurons that is not capsaicin sensitive (Kwan et al., 2006). Because the number of TRPA1-positive neurons detected by in situ hybridization was rather low, the discrepancy might be caused by differences in sensitivity of the techniques. There is strong data supporting the notion that TRPM8 mediates cold responses in vivo as well as in heterologous expression systems (McKemy et al., 2002; Peier et al., 2002). It has been shown to give rise to at least part of the innocuous cool sensation. Whether it is also in part responsible for the nociceptive cold response is unclear. The colocalization with TRPV1 reported here, along with the wide range of activation thresholds of menthol- and cold-sensitive native sensory neurons, is compatible with this view (McKemy et al., 2002) and in agreement with single-unit recordings of cutaneous cold-sensitive nociceptors that are menthol sensitive (Vastani, Maurer, and Koltzenburg, unpublished observations).

An interesting feature of TRPA1 development was its differential emergence among IB4-positive and -negative populations. This finding is compatible with the view that neurons with similar receptive properties can be generated in different sublineages at different developmental stages.

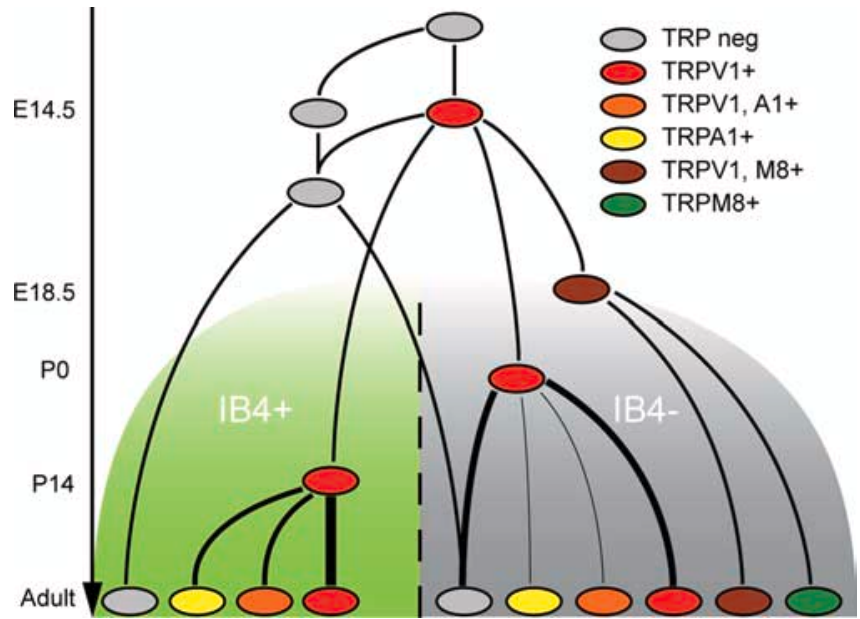

Figure 6. Proposed model of the developmental relationship between the different sensory neuron subtypes. AtE11.5, TRP-negative cells (gray) acquire TRPV1 (red) until the peak atE14.5, after which some cells will downregulate TRPV1. These cells give rise to the TRPV1-, IB4+ cells and some of the TRPV1 - IB4 - cells. Before birth, at E18.5, the cells expressing TRPM8 (green and brown) are specified from within the TRPV1-positive population. Some cells subsequently downregulate TRPV1, giving rise to two populations of TRPM8-positive neurons (with or without TRPV1; brown and green, respectively). Within the IB4-negative population, the TRPA1-positive cells derive from TRPV1-positive neurons perinatally, whereas within the IB4positive population, the TRPA1-expressing cells arise $>2$ weeks later. Some of the TRPA1- and TRPV1-coexpressing cells (orange) will also downregulate TRPV1, giving rise to TRPA1-only neurons (yellow). A1, TRPA1; M8, TRPM8; neg, negative.

\section{Lineage relationships}

A striking finding of the present study was that the menthol and capsaicin responsiveness completely overlapped during early development and that this overlap decrease just after birth. Because these changes occur when the percentage of menthol-sensitive neurons is relatively stable, it is plausible that this effect is caused by a specification of existing cells, losing the sensitivity to capsaicin, rather than a loss of the actual cells along with an induction of menthol sensitivity in a new set of cells. This hypothesis is supported by the finding that the severe loss of small-diameter sensory neurons with systemic neonatal capsaicin treatment also produces an equal reduction in heat-sensitive nociceptors and cold-sensitive (and menthol-sensitive) thermoreceptors (Welk et al., 1984). A similar finding was also seen in the development of mustard oil responsiveness, which was widely overlapping in the first two postnatal weeks. A mustard oil-sensitive, capsaicininsensitive population emerged after that time in the IB4-positive population. Similarly, eliminating TRPV1-expressing neurons at birth by systemic capsaicin treatment abolishes the response to mustard oil (Jancso et al., 1967; Holzer, 1991) at a developmental time when there is no expression of TRPA1, indicating that this population emerges from the perinatally TRPV1-expressing neurons. Based on the data presented in this study, we propose a model of how the identity of different classes of sensory neurons emerges during development (Fig. 6). Our data suggest that TRPM8-expressing neurons appear at E18.5 from the population of TRPV1-positive cells. Most of these neurons downregulate TRPV1 expression postnatally, giving rise to two populations: one still responsive to heat, expressing TRPV1, and the other, TRPM8 thermoreceptive neurons lacking TRPV1. However, none of them undergo the switch in neurotrophin dependency that is indicated by the upregulation of IB4. In this IB4-negative population, TRPA1 is present in TRPV1-positive neurons at P7, and a small group of neurons that is only TRPA1 positive is found 
in the adult. Interestingly, in the IB4-negative population, but not in the IB4-positive population, a majority of the TRPA1responsive neurons exhibit a cold sensitivity. Also in this IB4negative population, some of the neurons will lose their TRPV1 expression, giving rise to the peptidergic, non-heat-responsive nociceptors.

We conclude that in the mouse, the specification of peripheral sensory neurons that are derived from the truncal neural crest is a long and progressive process (supplemental Fig. 2, available at www.jneurosci.org as supplemental material). In the lineage of small-diameter sensory neurons that give rise to thermoreceptors and various kinds of nociceptors, the process of diversification starts at E12.5 and continues well into the adult life. Future research will need to address the question of whether this prolonged process can be found in other species and whether it is a general feature of sensory neurons of placodal origin.

\section{References}

Aquino J, Hjerling-Leffler J, Koltzenburg M, Edlund T, Villar M, Ernfors P (2006) In vitro and in vivo differentiation of boundary cap neural crest stem cells into mature Schwann cells. Exp Neurol 198:438-449.

Bandell M, Story GM, Hwang SW, Viswanath V, Eid SR, Petrus MJ, Earley TJ, Patapoutian A (2004) Noxious cold ion channel TRPA1 is activated by pungent compounds and bradykinin. Neuron 41:849-857.

Bautista DM, Movahed P, Hinman A, Axelsson HE, Sterner O, Hogestatt ED, Julius D, Jordt SE, Zygmunt PM (2005) Pungent products from garlic activate the sensory ion channel TRPA1. Proc Natl Acad Sci USA 102:12248-12252.

Bautista DM, Jordt SE, Nikai T, Tsuruda PR, Read AJ, Poblete J, Yamoah EN, Basbaum AI, Julius D (2006) TRPA1 mediates the inflammatory actions of environmental irritants and proalgesic agents. Cell 124:1269-1282.

Benn SC, Costigan M, Tate S, Fitzgerald M, WoolfCJ (2001) Developmental expression of the TTX-resistant voltage-gated sodium channels $\mathrm{Na}_{\mathrm{v}} 1.8$ (SNS) and $\mathrm{Na}_{\mathrm{v}} 1.9$ (SNS2) in primary sensory neurons. J Neurosci 21:6077-6085.

Bogen O, Dreger M, Gillen C, Schroder W, Hucho F (2005) Identification of versican as an isolectin B4-binding glycoprotein from mammalian spinal cord tissue. FEBS J 272:1090-1102.

Bouron A, Becker C, Porzig H (1999) Functional expression of voltagegated $\mathrm{Na}+$ and $\mathrm{Ca} 2+$ channels during neuronal differentiation of PC12 cells with nerve growth factor or forskolin. Naunyn Schmiedebergs Arch Pharmacol 359:370-377.

Buchman VL, Davies AM (1993) Different neurotrophins are expressed and act in a developmental sequence to promote the survival of embryonic sensory neurons. Development 118:989-1001.

Caterina MJ, Schumacher MA, Tominaga M, Rosen TA, Levine JD, Julius D (1997) The capsaicin receptor: a heat-activated ion channel in the pain pathway. Nature 389:816-824.

Caterina MJ, Leffler A, Malmberg AB, Martin WJ, Trafton J, Petersen-Zeitz KR, Koltzenburg M, Basbaum AI, Julius D (2000) Impaired nociception and pain sensation in mice lacking the capsaicin receptor. Science 288:306-313.

Clapham DE (2003) TRP channels as cellular sensors. Nature 426:517-524.

Dirajlal S, Pauers LE, Stucky CL (2003) Differential response properties of IB(4)-positive and -negative unmyelinated sensory neurons to protons and capsaicin. J Neurophysiol 89:513-524.

Felts PA, Yokoyama S, Dib-Hajj S, Black JA, Waxman SG (1997) Sodium channel alpha-subunit mRNAs I, II, III, NaG, Na6 and hNE (PN1): different expression patterns in developing rat nervous system. Brain Res Mol Brain Res 45:71-82.

Fitzgerald M, Gibson S (1984) The postnatal physiological and neurochemical development of peripheral sensory $\mathrm{C}$ fibres. Neuroscience 13:933-944.

Funakoshi K, Nakano M, Atobe Y, Goris RC, Kadota T, Yazama F (2006) Differential development of TRPV1-expressing sensory nerves in peripheral organs. Cell Tissue Res 323:27-41.

Holzer P (1991) Capsaicin: cellular targets, mechanisms of action, and selectivity for thin sensory neurons. Pharmacol Rev 43:143-201.

Inoue K, Ozaki S, Shiga T, Ito K, Masuda T, Okado N, Iseda T, Kawaguchi S, Ogawa M, Bae SC, Yamashita N, Itohara S, Kudo N, Ito Y (2002) Runx3 controls the axonal projection of proprioceptive dorsal root ganglion neurons. Nat Neurosci 5:946-954.

Jancso N, Jancso-Gabor A, Szolcsanyi J (1967) Direct evidence for neurogenic inflammation and its prevention by denervation and by pretreatment with capsaicin. Br J Pharmacol Chemother 31:138-151.

Jordt SE, McKemy DD, Julius D (2003) Lessons from peppers and peppermint: the molecular logic of thermosensation. Curr Opin Neurobiol 13:487-492.

Jordt SE, Bautista DM, Chuang HH, McKemy DD, Zygmunt PM, Hogestatt ED, Meng ID, Julius D (2004) Mustard oils and cannabinoids excite sensory nerve fibres through the TRP channel ANKTM1. Nature 427:260-265.

Julius D, Basbaum AI (2001) Molecular mechanisms of nociception. Nature 413:203-210.

Kaufman MH (1992) The atlas of mouse development. London: Academic. Kobayashi K, Fukuoka T, Obata K, Yamanaka H, Dai Y, Tokunaga A, Noguchi K (2005) Distinct expression of TRPM8, TRPA1, and TRPV1 mRNAs in rat primary afferent neurons with adelta/c-fibers and colocalization with trk receptors. J Comp Neurol 493:596-606.

Koltzenburg M (2004) Thermal sensitivity of sensory neurons. In: The pain system in normal and pathological states: a primer for clinicians (Villanueva L, Dickenson AH, Ollat H, eds), pp 29-43. Seattle: International Association for the Study of Pain.

Kwan KY, Allchorne AJ, Vollrath MA, Christensen AP, Zhang DS, Woolf CJ, Corey DP (2006) TRPA1 contributes to cold, mechanical, and chemical nociception but is not essential for hair-cell transduction. Neuron 50:277-289.

Lawson SN (2002) Phenotype and function of somatic primary afferent nociceptive neurones with C-, Adelta- or Aalpha/beta-fibres. Exp Physiol $87: 239-244$.

Lawson SN, Biscoe TJ (1979) Development of mouse dorsal root ganglia: an autoradiographic and quantitative study. J Neurocytol 8:265-274.

Leffler A, Mönter B, Koltzenburg M (2006) The role of the capsaicin receptor TRPV1 and acid-sensing ion channels (ASICS) in proton sensitivity of subpopulations of primary nociceptive neurons in rats and mice. Neuroscience 139:699-709.

Lin JH, Saito T, Anderson DJ, Lance-Jones C, Jessell TM, Arber S (1998) Functionally related motor neuron pool and muscle sensory afferent subtypes defined by coordinate ETS gene expression. Cell 95:393-407.

Marmigere F, Montelius A, Wegner M, Groner Y, Reichardt LF, Ernfors P (2006) The Runx1/AML1 transcription factor selectively regulates development and survival of TrkA nociceptive sensory neurons. Nat Neurosci 9:180-187.

McKemy DD, Neuhausser WM, Julius D (2002) Identification of a cold receptor reveals a general role for TRP channels in thermosensation. Nature 416:52-58.

Meyer RA, Ringkamp M, Campbell JN, Raja SN (2005) Peripheral mechanisms of cutaneous nociception. In: Wall and Melzack's textbook of pain (McMahon SB, Koltzenburg M, eds), pp 3-34. Edinburgh: Churchill Livingstone.

Molliver DC, Snider WD (1997) Nerve growth factor receptor TrkA is down-regulated during postnatal development by a subset of dorsal root ganglion neurons. J Comp Neurol 381:428-438.

Molliver DC, Wright DE, Leitner ML, Parsadanian AS, Doster K, Wen D, Yan Q, Snider WD (1997) IB4-binding DRG neurons switch from NGF to GDNF dependence in early postnatal life. Neuron 19:849-861.

Munns C, AlQatari M, Koltzenburg M (2007) Many cold sensitive peripheral neurons of the mouse do not express TRPM8 or TRPA1. Cell Calcium, in press.

Peier AM, Moqrich A, Hergarden AC, Reeve AJ, Andersson DA, Story GM, Earley TJ, Dragoni I, McIntyre P, Bevan S, Patapoutian A (2002) A TRP channel that senses cold stimuli and menthol. Cell 108:705-715.

Perl E (1992) Function of dorsal root ganglion neurons: an overview. In: Sensory neurons: diversity, development, and plasticity (Scott SA, ed), pp 3-23. New York: Oxford UP.

Pfaffl MW, Horgan GW, Dempfle L (2002) Relative expression software tool (REST) for group-wise comparison and statistical analysis of relative expression results in real-time PCR. Nucleic Acids Res 30:e36.

Priestley JV, Michael GJ, Averill S, Liu M, Willmott N (2002) Regulation of nociceptive neurons by nerve growth factor and glial cell line derived neurotrophic factor. Can J Physiol Pharmacol 80:495-505. 
Reid G (2005) ThermoTRP channels and cold sensing: what are they really up to? Pflügers Arch 451:250-263.

Silverman JD, Kruger L (1988) Lectin and neuropeptide labeling of separate populations of dorsal root ganglion neurons and associated "nociceptor" thin axons in rat testis and cornea whole-mount preparations. Somatosens Res 5:259-267.

Snider WD (1994) Functions of the neurotrophins during nervous system development: what the knockouts are teaching us. Cell 77:627-638.

Story GM, Peier AM, Reeve AJ, Eid SR, Mosbacher J, Hricik TR, Earley TJ, Hergarden AC, Andersson DA, Hwang SW, McIntyre P, Jegla T, Bevan S, Patapoutian A (2003) ANKTM1, a TRP-like channel expressed in nociceptive neurons, is activated by cold temperatures. Cell 112:819-829.

Tamura S, Morikawa Y, Senba E (2005) TRPV2, a capsaicin receptor homologue, is expressed predominantly in the neurotrophin-3dependent subpopulation of primary sensory neurons. Neuroscience 130:223-228.

Welk E, Fleischer E, Petsche U, Handwerker HO (1984) Afferent C-fibres in rats after neonatal capsaicin treatment. Pflügers Arch 400:66-71.

Williams S, Evan G, Hunt SP (1990) Spinal c-fos induction by sensory stimulation in neonatal rats. Neurosci Lett 109:309-314.

Woodbury CJ, Zwick M, Wang S, Lawson JJ, Caterina MJ, Koltzenburg M, Albers KM, Koerber HR, Davis BM (2004) Nociceptors lacking TRPV1 and TRPV2 have normal heat responses. J Neurosci 24:6410-6415.

Zwick M, Davis BM, Woodbury CJ, Burkett JN, Koerber HR, Simpson JF, Albers KM (2002) Glial cell line-derived neurotrophic factor is a survival factor for isolectin B4-positive, but not vanilloid receptor 1-positive, neurons in the mouse. J Neurosci 22:4057-4065. 